\title{
ASPECTOS DE LA DISTRIBUCIÓN LARVAL DE STOMATOPODA (CRUSTÁCEA) EN AGUAS SUPERFICIALES DEL PACÍFICO COLOMBIANO
}

\section{ASPECTS OF LARVAL DISTRIBUTION OF STOMATOPODA (CRUSTACEA) IN SURFACE WATERS OF THE COLOMBIAN PACIFIC}

\author{
Raúl H. López ${ }^{1}$, Juan C. Jaimes ${ }^{2}$ \\ ${ }^{1}$ Biólogo Marino, Dr. rer. nat. Profesor Asociado, e-mail: raul.lopez@unimilitar.edu.co; ${ }^{2}$ Biólogo Marino, M.Sc. Asistente de \\ Investigación, e-mail: juan.jaimes@unimilitar.edu.co, Universidad Militar Nueva Granada, Campus Nueva Granada, km 2 vía \\ Cajicá-Zipaquirá. Cundinamarca, Colombia.
}

Rev.U.D.C.A Act \& Div. Cient. 17(1): 227-236, Enero- Junio, 2014

\section{RESUMEN}

La distribución larval de estomatópodos en aguas superficiales del Pacífico colombiano, se evaluó alrededor de septiembre, entre el 2001 y 2004. Mediante una red cónica con poro de $363 \mu \mathrm{m}$, se obtuvieron 98 muestras superficiales de mesozooplancton, de las cuales, se extrajeron todas las larvas de estomatópodos. Se estimaron las abundancias para evaluar las posibles relaciones con la temperatura, la salinidad, la hora de captura, las fases lunares y la circulación superficial. Los promedios térmicos $\left(26,9^{\circ} \mathrm{C}\right)$ y salinos $(31,8$ UPS) indicaron condiciones normales en el área de estudio, con mayor influencia de la temperatura sobre la abundancia larval, cuyo promedio fue más alto en septiembre de 2004 (197 Ind./1000m ${ }^{3}$ ), que en los otros periodos (>27 Ind./ $\left.1000 \mathrm{~m}^{3}\right)$. Aunque se hallaron larvas, incluso hasta 556 $\mathrm{km}$ de la costa, en todos los periodos los mayores valores (70-2282 Ind./1000 $\left.\mathrm{m}^{3}\right)$, se registraron en aguas neríticas del centro-sur $\left(3-5^{\circ} \mathrm{N}\right)$, quizá en relación con el asentamiento de poblaciones adultas, en esa zona. Se observó una tendencia al aumento larvario en la noche, cuarto menguante y cuarto creciente. Es muy probable que en la distribución larval influya también la dinámica hidrológica del área de estudio.

Palabras clave: Stomatopoda, distribución larval, fases lunares, nictimeral, Pacífico colombiano.

\section{SUMMARY}

The distribution of stomatopod larvae in surface waters of the Colombian Pacific was evaluated around September of the years 2001-2004. By means of a conical $363 \mu \mathrm{m}$-pore net 98 surface mesozooplankton samples were obtained, of which all stomatopod larvae were extracted. Their abundances were estimated to evaluate possible relationships, with temperature, salinity, catch time, moon phases, and surface circulation. Thermal $\left(26.9^{\circ} \mathrm{C}\right)$ and saline (31.8 UPS) averages indicated normal conditions in the study area, with higher thermal influence on larval abundance, whose average was higher in $2004\left(197\right.$ Ind./1000m $\left.{ }^{3}\right)$ than in the other periods $\left(>27\right.$ Ind. $\left./ 1000 \mathrm{~m}^{3}\right)$. Although larvae were found even $556 \mathrm{~km}$ offshore, in all periods the highest values (70-2282 Ind./1000 $\left.\mathrm{m}^{3}\right)$ occurred in south-central neritic waters $\left(3-5^{\circ} \mathrm{N}\right)$, maybe related to the settlement of adult populations in that area. A trend in larval increasing by night, moon waning and moon waxing was observed. It is very likely that the hydrological dynamics of the study area has also an influence on larval distribution.

Key words: Stomatopoda, larval distribution, lunar phases, nictemeral, Colombian Pacific.

\section{INTRODUCCIÓN}

Los estomatópodos son crustáceos marinos y algunos estuarinos de las regiones tropicales, subtropicales y templadas, que habitan en el intermareal, en galerías y en grietas de rocas y de corales, hasta $1000 \mathrm{~m}$ de profundidad (Manning, 1995). En el mundo, se conocen cerca de 450 especies (Müller, 1994), de ellas, 53 especies y subespecies se han registrado en el Pacífico americano y sólo 11 especies, en el Pacífico colombiano (Murillo, 1988; Murillo \& Alvarez, 2004; Barragán \& Hendrickx, 2010). 
Como componente importante en los ecosistemas bentónicos, a veces abundante (Manning, 1995), la comunidad de estomatópodos es frecuente en las capturas de camarones (Hendrickx \& Sánchez, 2005) y langostinos (Roux \& Piñero, 2006). Varias especies son de importancia comercial (Vila et al. 2013).

Al igual que en peces, moluscos y otros crustáceos, las fases tempranas de los estomatópodos hacen parte del meroplancton, cuyo estudio da una mejor comprensión de procesos ecológicos fundamentales, como la dispersión/ retención, el reclutamiento y la conectividad (Cowen et al. 2007; Pineda et al. 2007; Cowen \& Sponaugle, 2009; Sponaugle et al. 2002); en ésta son más relevantes los estadios tempranos, pues los crustáceos adultos son sésiles o tienen poco desplazamiento (Gawarkiewicz et al. 2007; Siegel et al. 2008; Díaz et al. 2012). Las larvas de estomatópodos son también alimento representativo de varios peces, como ocurre en el Pacífico colombiano (Gómez et al. 1999, 2003; Navia et al. 2006) y en otras regiones del Pacífico americano, e.g., México (Valadez et al. 2001; Raymundo \& Saucedo, 2008) y Ecuador (Morales et al. 2004; Ramírez et al. 2008).

Los estomatópodos tienen de cuatro a nueve estadios larvales planctónicos durante varios meses (Reaka \& Manning, 1987), con un consecuente potencial de dispersión alto, lo que explica su rango latitudinal amplio (Díaz et al. 2012). Como en los demás organismos planctónicos (Harrison et al. 2004; Suthers \& Rissik, 2009; Mullaney \& Suthers, 2013), su dinámica está influenciada por un cuadro complejo de factores en acoplamiento físico-biológico, e.g., transporte pasivo o advectivo, atribuible a factores físicos, en particular las corrientes (Barber et al. 2002a, b; Lavaniegos et al. 2010), así como la topografía costera e insular, la migración vertical circadiana (nictimeral), las épocas de reproducción y las relaciones tróficas (Cowen et al. 2007; Gawarkiewicz et al. 2007; Díaz et al. 2012).

En el Pacífico americano, no se han efectuado estudios exclusivos sobre larvas de estomatópodos, sino que se incluyen en evaluaciones generales de zooplancton, e.g., en aguas de California (Jiménez et al. 2000, 2001; Lavaniegos et al. 2009, 2010), en Ecuador (Bonilla, 1983) y en Chile (Díaz et al. 2012). Lo mismo ocurre en el Pacífico colombiano, en donde sólo han sido considerados en cuatro publicaciones (Giraldo \& Gutiérrez, 2007; Martínez et al. 2007; Murcia \& Giraldo, 2007; Giraldo et al. 2011).

Lo anterior explica la realización de este trabajo. Además, los estudios ecológicos sobre los estadios tempranos y adultos de los estomatópodos son relevantes, por su rol en la trama trófica (Gómez et al. 1999, 2003; Navia et al. 2006; Vinuesa, 2005), la dispersión larvaria (Cowen \& Sponaugle, 2009; Sponaugle et al. 2002) y los hábitos carroñeros de los adultos, pues al consumir los organismos muertos descarta- dos por las flotas pesqueras, aceleran la recirculación de la materia orgánica (Vinuesa, 2005).

Se evalúa el efecto de la temperatura, la salinidad y la luminosidad (variación nictimeral) sobre su distribución y su abundancia. Aunque no se tomaron datos de la circulación superficial imperante en el Pacífico colombiano, también se analizó su posible influencia sobre la distribución larval. Esto se justifica, pues la dinámica hídrica es el principal factor de distribución del plancton, en general (Harrison et al. 2004; Suthers \& Rissik, 2009; Mullaney \& Suthers, 2013), incluidas, las larvas de estomatópodos (Barber et al. 2002a, b; Lavaniegos et al. 2010).

\section{MATERIALES Y MÉTODOS}

El Pacífico colombiano tiene $367.823 \mathrm{~km}^{2}$ y se localiza entre los $01^{\circ} 30^{\prime}$ y $07^{\circ} 10^{\prime}$ de latitud norte y los $77^{\circ} 40^{\prime}$ y $85^{\circ} 00^{\prime}$ longitud oeste (Fernández et al. 2003). De julio a septiembre impera un giro o circulación ciclónica elíptica relativamente central, de menor extensión y velocidad que en el resto del año. El noroeste del giro es influenciado por la Corriente del Golfo de Panamá, que fluye hacia el suroeste, mientras que en la zona costera, se desplaza de sur a norte la Corriente de Colombia, con mayor fuerza de mayo a diciembre (Figura 1). Todo el año, se manifiestan procesos de surgencia, pero con menor extensión e intensidad entre agosto y octubre, periodo con mayor estratificación de las masas de agua y una termoclina más profunda hacia la costa (CCCP, 2002; Rodríguez \& Schneider, 2003; Villegas, 2003).

En el marco del Estudio Regional del Fenómeno El Niño (ERFEN)-Colombia, se efectuaron cuatro campañas oceanográficas, designadas como 2001 a 2004, con 19, 20, 21 y 20 jornadas, respectivamente. Para facilitar la comparación con otros trabajos, se mantuvo la nomenclatura de las estaciones establecidas dentro del ERFEN, cuyo número, así como el derrotero del buque, son establecidos por la Dirección General Marítima (DIMAR) y el Centro Control de Contaminación del Pacífico (CCCP), para cada campaña oceanográfica, $i$. e., no es estándar (Figura 1, Tabla 1), así que no es posible que los muestreos se efectúen en igual número de estaciones nocturnas y diurnas, ni según la fase lunar. En los mapas de distribución se especifican las estaciones donde se encontraron larvas de estomatópodos.

Para obtener el zooplancton, en el primer metro superficial, se realizaron arrastres circulares de 10 minutos, a una velocidad de 3 nudos, con una red cónica $(\varnothing=50 \mathrm{~cm}$, longitud $1,85 \mathrm{~m}$ y poro $363 \mu \mathrm{m})$, a la cual, se fijó un flujómetro General Oceanics, para determinar el volumen de agua filtrada. Las muestras, se fijaron con formaldehido al $10 \%$ en agua de mar. Se empleó una sonda multiparamétrica perfiladora CTD Seabird-19, para registrar la temperatura y la salinidad. 


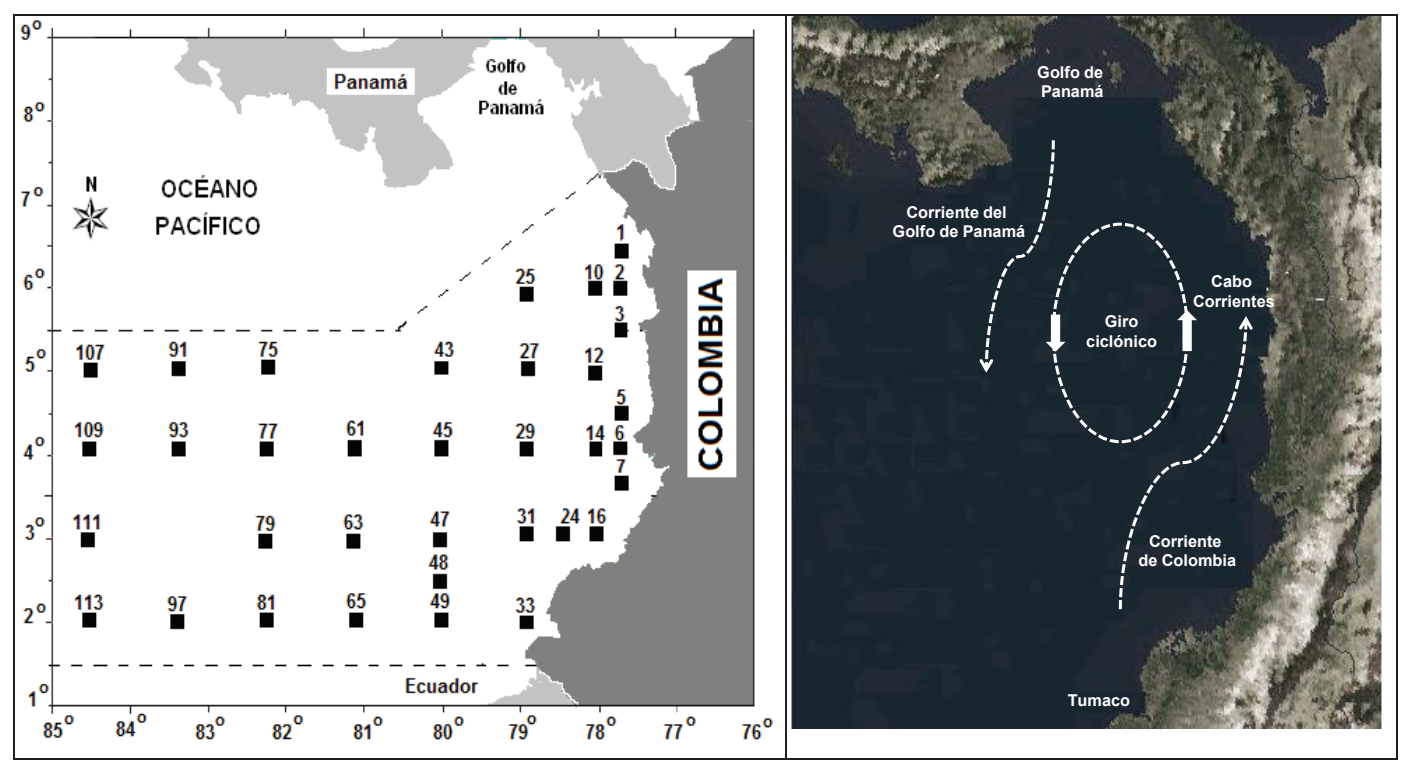

Figura 1. Mapa del Pacífico colombiano, estaciones ERFEN-Colombia, para la captura de mesozooplancton en los cinco periodos de estudio y principales corrientes superficiales.

Tabla 1. Número de estaciones por campaña oceanográfica en el Pacífico colombiano, durante los cuatro periodos de estudio. Se especifica el número de estaciones nocturnas, diurnas y por fase lunar.

\begin{tabular}{|c|c|c|c|c|c|c|}
\hline \multirow{2}{*}{\multicolumn{2}{|c|}{ Campaña oceanográfica }} & 2001 & 2002 & 2003 & 2004 & \multirow{2}{*}{ Total } \\
\hline & & 28.Ago-15.Sep. & 03-22.Sep. & 01-21.Sep. & 19.Sep-08.Oct. & \\
\hline \multicolumn{2}{|c|}{ Jornadas } & 19 & 20 & 21 & 20 & 80 \\
\hline \multirow{4}{*}{ Fase lunar } & Nueva & 0 & 6 & 4 & 4 & 14 \\
\hline & Creciente & 6 & 13 & 10 & 12 & 41 \\
\hline & Llena & 10 & 4 & 8 & 4 & 26 \\
\hline & Menguante & 7 & 2 & 3 & 5 & 17 \\
\hline \multirow{3}{*}{ Estaciones } & Total & 23 & 25 & 25 & 25 & 98 \\
\hline & Noche & 13 & 12 & 12 & 18 & 55 \\
\hline & Día & 10 & 13 & 13 & 7 & 43 \\
\hline
\end{tabular}

Con la ayuda de un estereoscopio Leica E4-Z10, se revisaron las 93 muestras obtenidas en los cuatro periodos de estudio y se extrajeron y contaron todas las fases larvarias de estomatópodos. Sus abundancias, se estandarizaron a 1000 $\mathrm{m}^{3}$ (Suthers \& Rissik, 2009), así: $\mathrm{N}=1000 \mathrm{~m}^{3} * \mathrm{n} / \pi^{*} \mathrm{r}^{2} * \mathrm{~d}$, donde: $\mathrm{N}=$ Número de individuos $/ 1000 \mathrm{~m}^{3}, \mathrm{n}=$ número de individuos en cada arrastre, $r=$ radio de la red, $d=$ distancia recorrida por la red en cada arrastre, $\pi * r^{2} * d=$ volumen de agua filtrada en cada arrastre. La distribución por periodo de estudio, se ilustró en mapas elaborados con el programa Surfer $8 \AA$.

Dada la migración vertical de las larvas de crustáceos, debida al cambio en las condiciones de luz (Díaz et al. 2012), se incluyeron análisis comparativos día : noche; las muestras obtenidas de las 6:00 a las 18:00 h se denominaron diurnas y de las 18:01 a las 05:59 h nocturnas. Además, se consideraron las fases de luna llena, cuarto menguante, luna nueva y cuarto creciente.

Los datos de abundancia, se transformaron con $\log (x+1)$, a fin de reducir el sesgo debido a los valores más elevados pues, según la prueba de Shapiro-Wilk, los datos no tenían una distribución normal. Se evaluó la relación entre las abundancias, la salinidad (UPS = Unidades Prácticas de Salinidad), la temperatura $\left({ }^{\circ} \mathrm{C}\right)$, la hora de captura y las fases lunares, mediante un Análisis Factorial por componentes principales, por periodo y aplicado al conjunto general de datos. Para facilitar la explicación, se efectuó una rotación ortogonal varimax de los factores, i.e., una normalización, 
sustrayendo las medias de los valores de las variables y dividiendo por sus desviaciones estándar. El Análisis Factorial incluye las comunalidades, correspondientes al porcentaje de variabilidad estimado en cada variable; entre más alta es la comunalidad es mayor la representación de las variables en los factores. El análisis estadístico, se realizó con STATGRAPHICS Centurión XVI®.

Luego, se realizó una prueba de Kruskall-Walls (K-W), con el fin de evidenciar alguna diferencia entre los cuatro periodos evaluados, en relación con la temperatura, la salinidad y las abundancias larvales de estomatópodos y para determinar la incidencia de la variación nictimeral y las fases lunares sobre la abundancia larvaria.

\section{RESULTADOS Y DISCUSIÓN}

Temperatura y salinidad en la superficie: Los promedios de temperatura $\left({ }^{\circ} \mathrm{C}\right)$ y de salinidad (UPS) fueron: 27,3 y 31,7 , en 2001; 27,2 y 32,1, en 2002; 27,5 y 31,9, en 2003 y, 26,3 y 31,1, en 2004 (Figura 2). No hubo diferencias significativas entre los cuatro periodos (K-W: $\mathrm{p} \geq 0.05$ ), para estas dos variables.

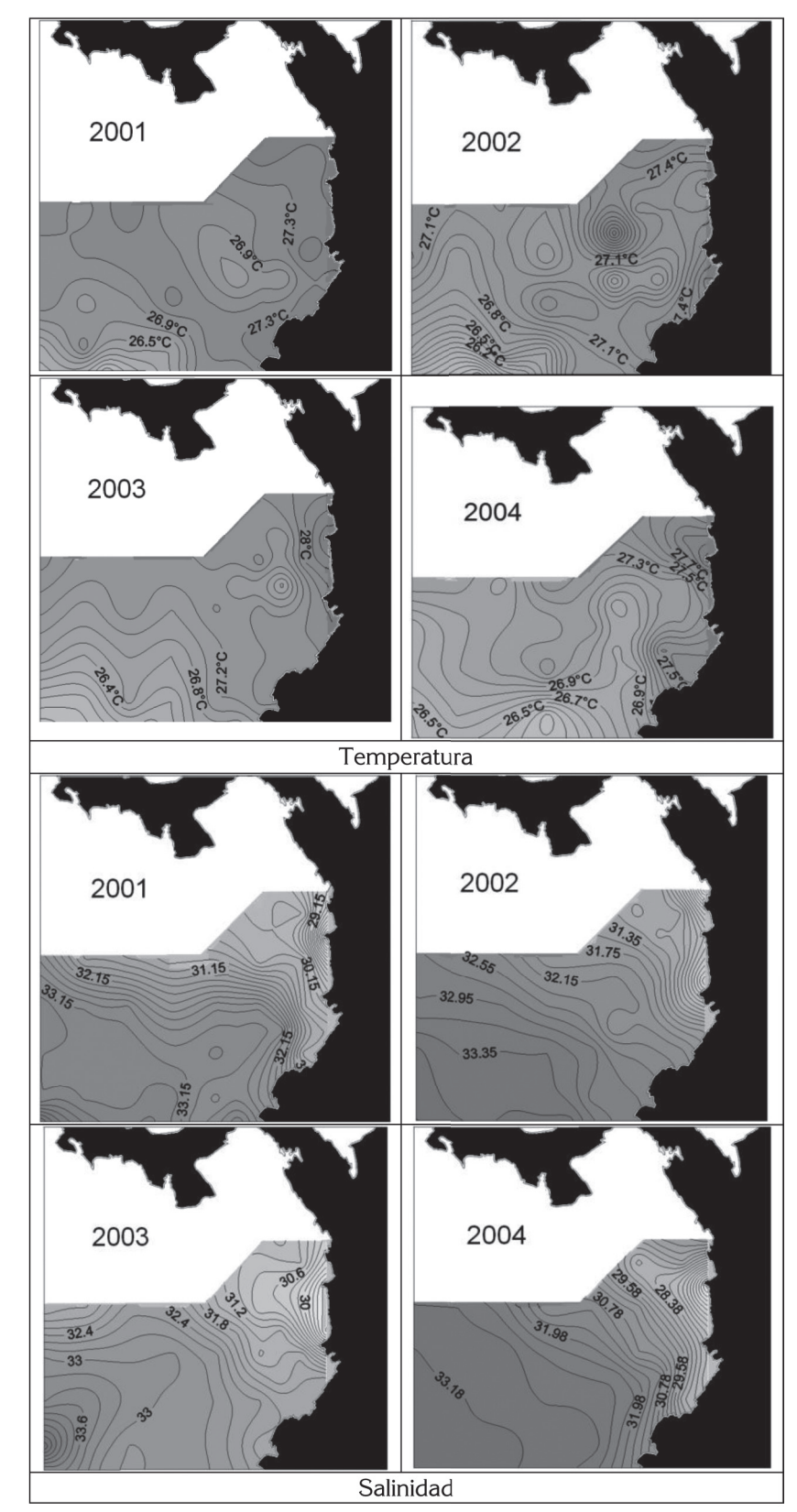

Figura 2. Temperatura $\left({ }^{\circ} \mathrm{C}\right)$ y salinidad superficiales (UPS) en el Pacífico colombiano, durante los cuatros periodos de estudio. 
En términos generales, la topografía dinámica superficial del Pacífico colombiano correspondió a la propia del segundo periodo del año, caracterizada por aguas del noreste, ligeramente más cálidas que las del suroeste, un gradiente de mayor a menor temperatura de la zona costera a la oceánica y un comportamiento inverso de la salinidad, con valores dentro de los rangos normales, reportados para el área de estudio (CCCP, 2002).

Abundancia larvaria de estomatópodos en la superficie: Puesto que los estadios postlarvarios de los estomatópodos se asientan (Morgan \& Provenzano, 1979; Díaz et al. 2012), en este trabajo, se consideró que solamente se capturaron larvas. Su abundancia promedio (Ind./1000 $\mathrm{m}^{3}$ ), en 2004, fue la más alta (197), seguida de 2002, (26), 2003 (9) y 2001, (9). Estas diferencias fueron corroboradas estadísticamente $(\mathrm{K}-\mathrm{W}: \mathrm{p}<0.05)$.

Los valores anteriores están dentro de lo esperado, pues las larvas de estomatópodos son poco numerosas en las muestras de zooplancton (Gawarkiewicz et al. 2007; Siegel et al. 2008; Díaz et al. 2012), como se ha observado también en el Pacífico colombiano, por Giraldo \& Gutiérrez (2007), Martínez et al. (2007), Murcia \& Giraldo (2007) y Giraldo et al. (2011), así como en otras áreas, e. g., Corriente de California (Jiménez et al. 2000, 2001; Lavaniegos et al. 2009, 2010), alrededores de las Islas Galápagos (Bonilla, 1983) y zona costera de Chile (Díaz et al. 2012).

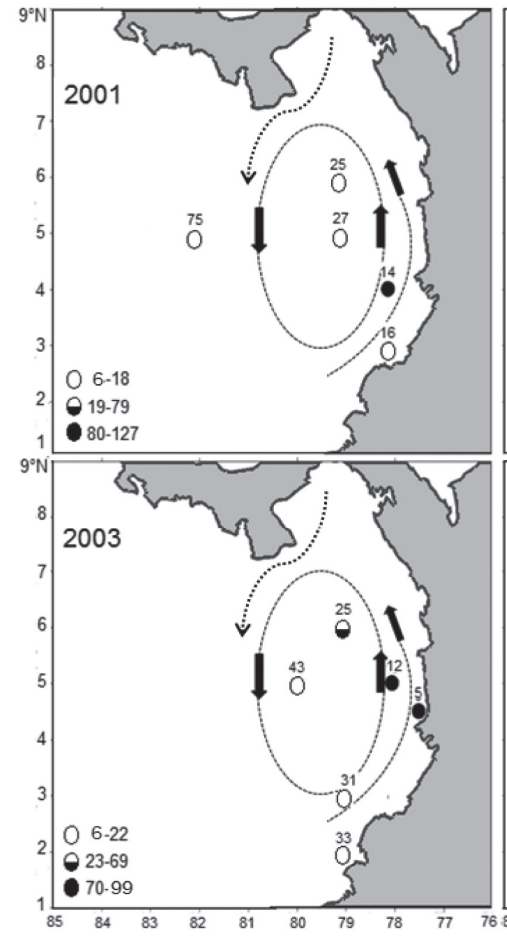

El bajo número se ha explicado por el ocultamiento diurno de las larvas en rocas o en escombros del fondo y su migración a la columna y superficie del agua al anochecer y amanecer, lo que reduce la exposición a las corrientes (Reaka et al. 2008). Este mecanismo es utilizado, incluso, para el asentamiento (Morgan \& Provenzano, 1979; Díaz et al. 2012), en conjunción con el régimen mareal; si las larvas son llevadas por la marea baja a un hábitat inadecuado, con la marea alta la migración vertical puede permitir su salida y posterior advección costera, hasta el ambiente apropiado para el asentamiento (Cowen et al. 2007; Gawarkiewicz et al. 2007; Díaz et al. 2012).

En los cuatro periodos, las mayores abundancias ( $>70$ Ind. $/ 1000 \mathrm{~m}^{3}$ ) se registraron entre los 3,0 y $5,5^{\circ} \mathrm{N}$ de la zona nerítica (Figura 3). Esto se podría relacionar con las poblaciones adultas que habitan los extensos bosques de manglar, fondos fangosos, fango-arenosos y sustratos duros en la isla Gorgona (CCCP, 2002), todos hábitats típicos de las once especies reportadas para el Pacífico colombiano; de hecho, en esa zona de gran importancia pesquera, se capturan estomatópodos adultos en los arrastres de camarón (Murillo, 1988; Fernández et al. 2003; Murillo \& Álvarez, 2004; Barragán \& Hendrickx, 2010).

En contraste, las abundancias larvarias de estomatópodos en todos los periodos fueron más bajas (6-211 Ind./1000m³)

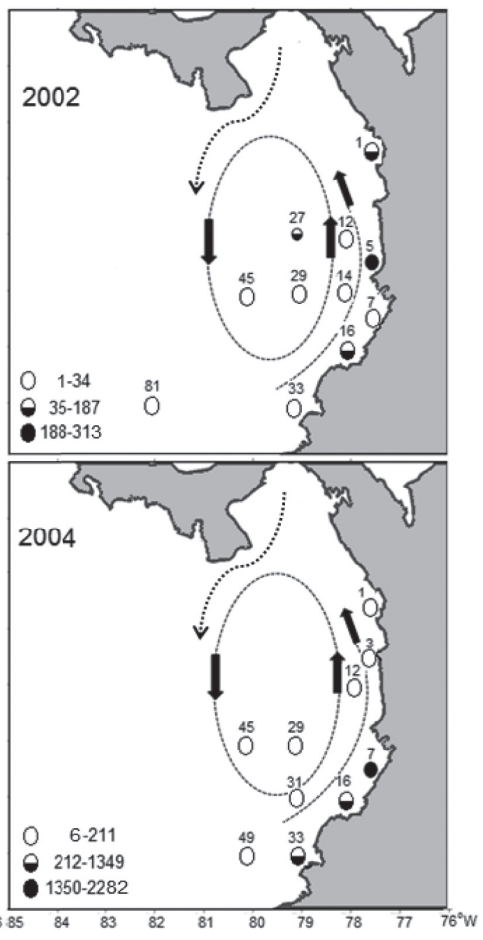

Figura 3. Distribución y abundancia larval de estomatópodos (Ind./1000 $\mathrm{m}^{3}$ ) en aguas superficiales del Pacífico colombiano, durante los cuatro periodos de estudio. El esquema de las corrientes del Golfo de Panamá, el giro ciclónico y la Corriente de Colombia muestra su posible influencia en el transporte superficial de las larvas hacia aguas costeras. 
hacia la zona de transición nerítica-oceánica; en aguas oceánicas, solamente se hallaron en las estaciones 75 (6 Ind./1000m ${ }^{3}$ ), en 2001 y 81 (1 Ind./1000m³), en 2002 (Figura 3).

En la misma área de estudio, para septiembre-octubre de 2004, Murcia \& Giraldo (2007) reportaron una distribución larvaria de estomatópodos similar a la del presente trabajo, mientras que en marzo de 2006, Martínez et al. (2007) observaron la mayor concentración en el norte del Pacífico colombiano, acaso, en parte, porque en esa época las condiciones ambientales y oceanográficas cambian, por el desplazamiento de la Zona de Convergencia Intertropical (CCCP, 2002).

Variación nictimeral y entre fases lunares de la abundancia larvaria promedio de estomatópodos: Se evidenciaron mayores capturas durante la noche (Ind./1000m³): 2001(11), 2002(44), 2003(13), 2004(262), que durante el día: 2001(3), 2002(9), 2003(7), 2004(2), aunque sin diferencias significativas (K-W: $\mathrm{p} \geq 0.05)$, seguramente, debido al bajo número de datos. Un problema importante en la Oceanografía biológica es la escasez de datos, sobre todo en el Pacífico colombiano, por lo cual, en otras partes del mundo se están desarrollando sistemas de simulación y observación de los océanos para cubrir esta necesidad (Lin et. al. 2010).

En el Pacífico colombiano, las mareas pueden alcanzar hasta $5 \mathrm{~m}$ en la zona central costera (IDEAM, 2012), i.e., entre los $3.0-5.5^{\circ} \mathrm{N}$, en donde la presencia de estadios tempranos de estomatópodos fue más representativa, según se anotó con anterioridad.

En relación con las fases lunares, las mayores abundancias promedio y frecuencias de captura fueron en cuarto creciente (264 Ind./1000m ${ }^{3}$; 0,41) y cuarto menguante (87 Ind./1000m ${ }^{3}$; 0,33) y las más bajas en luna llena y luna nueva (7 Ind./1000 $\mathrm{m}^{3}$; 0,25 en ambos casos). No se obtuvieron diferencias significativas (K-W: $\mathrm{p} \geq 0.05)$.

Bonilla (1983) también refirió alta abundancia en cuarto menguante en los alrededores de las Islas Galápagos y resaltó que un número elevado de larvas no es proporcional al número de estaciones, i.e., que se pueden capturar muchas, incluso, en pocos sitios. Reaka (1976) señaló algo similar y que la periodicidad lunar y mareal influyen en la reproducción de los estomatópodos y, por ende, en el número de larvas en el medio, según la época

Correlación entre variables: El análisis factorial mostró mayores asociaciones entre la abundancia $(0,67$ a 0,92), la temperatura $(-0,69$ a $-0,90)$ y la salinidad $(0,60$ a 0,82$)$, indicativo de la mayor influencia termohalina sobre la abundancia larval y la poca amplitud de sus rangos en la superficie. Tam- bién hubo alguna influencia inversa de la luz sobre la abundancia larval, evidenciada por las correlaciones altas con las fases lunares (-075 a 0,90; excepto en $2003=0,03)$ y la hora de captura (día : noche) $(0,64$ a 0,88$)$, lo que se interpretó como una influencia importante de la luz. Se consideraron los dos primeros componentes del análisis factorial (factores 1 y 2), porque en los cuatro periodos explicaron entre 65,04 y $68,54 \%$ de la variabilidad total de los datos (Tabla 2).

Al aplicar la prueba estadística a la totalidad de los datos, se obtuvieron mayores correlaciones para la abundancia $(0,75)$, la temperatura $(-0,67)$ y la fase lunar $(0,60)$; para la salinidad fue 0,30 y para la hora -0,27. Es probable que cuarto creciente y cuarto menguante fueron más favorables, dadas las abundancias larvales promedio más altas, durante esas fases lunares. Las correlaciones entre las variables, la gran mayoría positivas, mostraron cambios por periodo de estudio, atribuibles, en primer lugar, a la gran dinámica hídrica del Pacífico colombiano.

Distribución larvaria de estomatópodos y circulación superficial: Otro elemento asociado con la distribución larvaria de estomatópodos en los cuatro periodos estudiados, se atribuye al transporte por las corrientes. La distribución de las larvas sugiere que la Corriente del Golfo de Panamá, el giro ciclónico central y la Corriente de Colombia favorecen su transporte hacia aguas costeras. Lo dicho, se basa en que la mayor dispersión larval por la circulación macro-escalar (Cowen et al. 2007; Reaka et al. 2008; Cowen \& Sponaugle, 2009), se ha comprobado en muchas otras regiones, e.g., California (Jiménez et al. 2000, 2001; Lavaniegos et al. 2009, 2010), Ecuador (Bonilla, 1983) y Chile (Díaz et al. 2012).

La estocasticidad a escala media complica entender el rol y la dispersión/retención del meroplancton en aguas neríticas (Siegel et al. 2008). De todas maneras, de la oceanografía meso-escalar costera en el Pacífico colombiano, solamente se han referido algunos anillos o remolinos ciclónicos y anticiclónicos en la Corriente de Colombia (Alvariño, 1978) y variaciones mareales de ca. 5m (IDEAM, 2012). En otras corrientes costeras, tal circulación se asocia con la generación de focos de alta productividad y abundancia de zooplancton, en general (Harrison et al. 2004; Mullaney \& Suthers, 2013), en donde, inclusive, concurren aglomeraciones de larvas de estomatópodos, especialmente, en aguas tropicales, donde los adultos son más abundantes (Morgan \& Provenzano, 1979; Díaz et al. 2012).

Barber et al. (2002a, b) indican que las larvas de estomatópodos se alejan hasta unos $111 \mathrm{~km}$ de la costa, para facilitar su retorno o acceso a nuevas áreas, lo que, parcialmente, ayudaría a explicar la tendencia observada en el Pacífico colombiano a encontrar pocas larvas en aguas oceánicas (has- 
Tabla 2. Correlaciones entre las variables abióticas y la abundancia larval de estomatópodos en el Pacífico colombiano, durante los cuatro periodos de estudio y, en general, obtenidas mediante el análisis factorial (extracción por componentes principales).

\begin{tabular}{|c|rcc|ccc|}
\hline \multirow{3}{*}{ Variable } & \multicolumn{3}{|c|}{$2001 \mathrm{n}=23$} & \multicolumn{3}{c|}{$2002 \mathrm{n}=25$} \\
\cline { 2 - 7 } & \multicolumn{3}{|c|}{ Factor } & \multirow{2}{*}{ Comunalidad } & \multicolumn{3}{c|}{ Factor } & Comunalidad \\
& 1 & 2 & & \multicolumn{1}{c|}{2} & \\
\hline $\mathrm{n}$ & 0,93 & 0,05 & 0,86 & 0,80 & $-0,11$ & 0,65 \\
$\mathrm{~S}$ & 0,75 & $-0,06$ & 0,57 & 0,71 & 0,53 & 0,79 \\
$\mathrm{~T}^{\circ} \mathrm{C}$ & $-0,83$ & $-0,12$ & 0,70 & $-0,87$ & 0,13 & 0,78 \\
Día:noche & 0,04 & 0,78 & 0,60 & 0,09 & 0,74 & 0,56 \\
Fase lunar & 0,02 & 0,76 & 0,58 & 0,27 & $-0,76$ & 0,65 \\
\hline
\end{tabular}

\begin{tabular}{|c|rcc|ccc|}
\hline \multirow{3}{*}{ Variable } & \multicolumn{3}{|c|}{$2003 \mathrm{n}=25$} & \multicolumn{3}{c|}{$2004 \mathrm{n}=25$} \\
\cline { 2 - 7 } & \multicolumn{3}{|c}{ Factor } & \multirow{2}{*}{ Comunalidad } & \multicolumn{3}{|c|}{ Factor } & Comunalidad \\
& 1 & 2 & & 2 & \\
\hline $\mathrm{n}$ & 0,67 & $-0,44$ & 0,65 & 0,76 & 0,11 & 0,59 \\
$\mathrm{~S}$ & 0,60 & 0,48 & 0,60 & 0,82 & 0,04 & 0,68 \\
$\mathrm{~T}^{\circ} \mathrm{C}$ & $-0,90$ & 0,15 & 0,84 & $-0,69$ & $-0,49$ & 0,72 \\
Día:Noche & $-0,10$ & 0,88 & 0,79 & 0,19 & 0,64 & 0,45 \\
Fase lunar & 0,67 & 0,03 & 0,45 & 0,02 & 0,90 & 0,82 \\
\hline
\end{tabular}

\begin{tabular}{|c|c|c|c|}
\hline \multicolumn{4}{|c|}{ GENERAL $\mathrm{n}=98$} \\
\hline \multirow{2}{*}{ Variable } & \multicolumn{2}{|c|}{ Factor } & \multirow{2}{*}{ Comunalidad } \\
\hline & 1 & 2 & \\
\hline $\mathrm{n}$ & 0,75 & $-0,23$ & 0,61 \\
\hline S & 0,31 & 0,73 & 0,63 \\
\hline $\mathrm{T}^{\circ} \mathrm{C}$ & $-0,67$ & $-0,52$ & 0,72 \\
\hline Día:Noche & $-0,27$ & 0,75 & 0,64 \\
\hline Fase lunar & 0,60 & 0,10 & 0,36 \\
\hline
\end{tabular}

ta ca. 556km de la costa) y un número más alto y con mayor frecuencia hacia la zona costera.

En síntesis, los resultados muestran que la distribución larvaria en el Pacífico colombiano depende de las condiciones oceanográficas y la influencia de la luz y sugieren que también influye el comportamiento reproductivo y la ontogenia particular de cada especie, en el marco del acoplamiento físico-biológico, referido por otros autores (Barber et al. 2002a, b; Cowen et al. 2007; Gawarkiewicz et al. 2007; Lavaniegos et al. 2010; Díaz et al. 2012).
Dada la dificultad para evaluar la dispersión/retención y diversidad de los estomatópodos (Reaka \& Manning, 1987; Reaka et al. 2008), los estudios poblacionales, filogenéticos y filogeográficos se refuerzan con análisis moleculares de larvas y adultos (Barber et al. 2002a, b; Barber \& Boyce, 2006). Además, la identificación de los estadios larvarios es mucho más difícil que la de los adultos, también complicada (Müller, 1994; Tang et al. 2010). Por esto, no se establecieron las identidades de los ejemplares capturados en el Pacífico colombiano que, además, se fijaron y se conservaron con formaldehído, el cual, desnaturaliza el ADN; sin embargo, es importante anotar que pudieron corresponder a algunas 
de las siguientes tres familias y 11 especies, registradas al presente para el Pacífico colombiano, en especial, en la zona central-sur $\left(3 \cdot 0-5 \cdot 5^{\circ} \mathrm{N}\right)$, donde se halló la mayor abundancia y frecuencia larvaria de estomatópodos: Gonodactylidae: Gonodactylus zacae, G. festae, G. albicinctus, G. bahiahondensis; Squillidae: Cloridopsis dubia, Squilla aculeata aculeata, S. biformis, S. mantoidea, S. parva, S. panamensis, Pseudosquillidae: Pseudosquilla adiastalta (Murillo, 1988; Murillo \& Álvarez, 2004; Barragán \& Hendrickx, 2010).

Conflictos de intereses: El manuscrito fue preparado y revisado con la participación de los dos autores, quienes declaramos que no existe ningún conflicto de intereses que ponga en riesgo la validez de los resultados presentados. Financiación: Este trabajo fue posible gracias a la financiación de Universidad Militar Nueva Granada y al apoyo logístico de la Dirección General Marítima (DIMAR), entidad que proporcionó el buque oceanográfico para la realización de los cruceros oceanográficos y el Centro Control de Contaminación del Pacífico (CCCP), el cual, suministró los datos crudos de temperatura y salinidad.

\section{BIBLIOGRAFÍA}

1. ALVARIÑO, A. 1978. El zooplancton del Pacífico colombiano y las pesquerías. Memorias. I Seminario de Oceanografía del Pacífico Sudeste. Septiembre 1-5/76, Cali. p.206-271.

2. BARBER, P.H.; MOOSA, M.K.; PALUMBI, S.R. 2002a. Rapid recovery of genetic diversity of stomatopod populations on Krakatau: temporal and spatial scales of marine larval dispersal. Proc. Biol. Sci. 269(1500):1591-1597.

3. BARBER, P.H.; PALUMBI, S.R.; ERDMANN, M.V.; MOOSA, M.K. 2002b. Sharp genetic breaks among populations of Haptosquilla pulchella (Stomatopoda) indicate limits to larval transport: patterns, causes, and consequences, Mol. Eco. 11:659-674.

4. BARBER, P.H.; BOYCE, S.L. 2006. Estimating diversity of Indo-Pacific coral reef stomatopods through DNA barcoding of stomatopod larvae. Proc. Biol. Sci. 273(1597):2053-2061.

5. BARRAGÁN, J.S.; HENDRICKX, M.E. 2010. Clave ilustrada para la identificación de los estomatópodos (Crustacea: Hoplocarida) del Pacífico oriental. Rev. Mex. Biol. 81:1-49.
6. BONILLA, D. 1983. El zooplancton de las Islas Galápagos. Acta Oceanogr. Pac. 2(1):119-146.

7. CCCP. 2002. Compilación oceanográfica de la Cuenca Pacífica Colombiana. Centro Control de Contaminación del Pacífico. Imágenes de la Naturaleza, Cali. 109p.

8. COWEN, R.K.; GAWARKIEWICZ, G.; PINEDA, J.; THORROLD, S.R.; WERNER, F.E. 2007. Population connectivity in marine systems: an overview. Oceanography 20:14-21.

9. COWEN, R.K.; SPONAUGLE, S. 2009. Larval dispersal and marine population connectivity. Ann.. Rev. Mar. Sci. 1:443-466.

10. DÍAZ, E.; HERNÁNDEZ, E.; HERNÁNDEZ, C.E.; QUIÑONES, R.A. 2012. Mesoscale $\beta$ diversity and spatial nestedness of crustacean larvae in the coastal zone off central southern Chile: population and community implications. ICES J. Mar. Sci. 69:429438.

11. FERNÁNDEZ, C.E.; ANGULO, J.A.; NEWMARK, F.; MOSQUERA, C. 2003. Una visión de pesca multiespecífica en el Pacífico colombiano. Adaptaciones tecnológicas. Ser. Doc. Gen. INVEMAR. 14:1-75.

12. GAWARKIEWICZ, G.; MONISMITH, S.; LARGIER, J. 2007. Observing larval transport processes affecting population connectivity: progress and challenges. Oceanography 20: 40-53.

13. GIRALDO, A.; GUTIÉRREZ, E. 2007. Composición taxonómica del zooplancton superficial en el Pacífico colombiano (septiembre 2003). Invest. Mar. 35(1):117-122.

14. GIRALDO, A.; VALENCIA, B.; RAMÍREZ, D.G. 2011. Productividad planctónica y condiciones oceanográficas locales en isla Gorgona durante julio de 2006. Bol. Investig. Mar. Costeras. 40(1):185201.

15. GÓMEZ, G.; ZAPATA, L.; FRANKE, R.; RAMOS, G. 1999. Hábitos alimentarios de Epinephelus acanthistius y notas de otros peces serránidos capturados en el Parque Nacional Natural Gorgona, Pacífico colombiano. Bol. Investig. Mar. Costeras. 28:43-60.

16. GÓMEZ, G.; ZAPATA, L.; FRANKE, R.; RAMOS, G. 2003. Hábitos alimentarios de Mustelus lunulatus y $M$. 
henlei (Pisces: Triakidae) colectados en el parque nacional natural Gorgona, Pacífico colombiano. Bol. Investig. Mar. Costeras. 32:219-229.

17. HARRISON, P.A.; WHITNEY, F.A.; TSUDA, A.; SAITO, H.; TADOKORO, K. 2004. Nutrient and plankton dynamics in the NE and NW gyres of the Subarctic Pacific Ocean. J. Oceanogr. 60:93-117.

18. HENDRICKX, M.; SÁNCHEZ, P. 2005. Estomatópodos del género Squilla (Haplocarida: Stomatopoda: Squillidae) recolectados frente a las costas de Sinaloa, en el SE del Golfo de California, México, en los cruceros CEEMEX C1-C2-C3. Cienc. Mar. 31(1A):31-41.

19. IDEAM. 2012. Pronóstico de pleamares y bajamares en la costa pacífica colombiana. Año 2013. Inst. Hidrol, Meteo. Est. Amb., Bogotá D.C. 130p.

20. JIMÉNEZ, L.C.; LAVANIEGOS, B.E.; BAUMGARTNER, T.R. 2000. Biomasa y estructura del zooplancton en la parte sur de la Corriente de California durante julio de 1998. Crucero IMECOCAL 9807. B/O Francisco de Ulloa. 15-30 de julio de 1998. Inf. Téc. Com. Acad. Ser. Ecol. CICESE, Ensenada, B. California, México. 41p.

21. JIMÉNEZ, L.C.; LAVANIEGOS, B.E.; BAUMGARTNER, T.R. 2001. Biomasa y estructura del zooplancton colectado por el crucero IMECOCAL 9809/10 en la parte sur de la Corriente de California. Inf. Téc. Com. Acad. Ser. Ecol. CICESE, Ensenada, B. California, México. 39p.

22. LAVANIEGOS, B.E.; CADENA, J.L.; GARCÍA, P.G. 2009. Biomasa y estructura del zooplancton frente a la costa occidental de Baja California durante 2006 (cruceros IMECOCAL 0602, 0604, 0607). Inf. Téc. Cen. Inv. Cient. y Ed. Sup.Ensenada (CICESE), Ensenada, B. California, México. 50p.

23. LAVANIEGOS, B.E.; AMBRIZ, I.; HEREU, C.; JIMÉNEZ, L.C.; CADENA, J.L.; GARCÍA, P. 2010. Variabilidad estacional e interanual del zooplancton. En: Gaxiola G.; Durazo, R. eds. Dinámica del ecosistema pelágico frente a Baja California, 1997-007: Diez años de investigaciones mexicanas de la Corriente de California, p.87-126.

24. LIN, P.; JI, R.; DAVIS, C.S.; MCGILLICUDDY, D.J. 2010. Optimizing plankton survey strategies using observing system simulation experiments. J. Mar. Syst. 82:187-194.
25. MANNING, R.B. 1995. Stomatopod Crustacea of Vietnam: The legacy of Raoul Serène. Crustacean Research, Car. Soc. Jap. Sp. Num. 4:1-339.

26. MARTÍNEZ, T.; GIRALDO, A; RODRÍGUEZ, E. 2007. Zooplancton en la Corriente de Colombia, Pacífico colombiano, durante marzo de 2006. Bol. Cient. CCCP 14:69-82.

27. MORALES, O.; CASTILLO, J.; SOSA, O.; RODRÍGUEZ, C. 2004. Catálogo de tiburones, rayas y quimeras (Chondrichthyes) que habitan en las aguas del norte del Golfo de California. Rep. Téc. Lab. Ecol. Pesq., CICESE, Ensenada, Baja California, México. 119p.

28. MORGAN, S.G.; PROVENZANO, A.J. 1979. Development of pelagic larvae and postlarva of Squilla empusa (Crustacea: Stomatopoda) with an assessment of larval characters within the Squillidae. Fishery Bull. 77(1):61-90.

29. MULLANEY, T.J.; SUTHERS, I.M. 2013. Entrainment and retention of the coastal larval fish assemblage by a short-lived, submesoscale, frontal eddy of the East Australian Current. Limnol. Oceanogr. 58(5):15461556.

30. MÜLLER, H.G. 1994. World catalogue and bibliography of the recent Stomatopoda. Laboratory for Tropical Ecosystems Research \& Information Service. Wis. Ver., Wetzlar, 312p.

31. MURCIA, M.; GIRALDO, A. 2007. Condiciones oceanográficas y composición del mesozooplancton en la zona oceánica del Pacífico colombiano, durante septiembre-octubre de 2004. Bol. Cient. CCCP.14:83-94.

32. MURILLO, C.B. 1988. Estomatópodos de la costa pacífica de Colombia e isla Gorgona (Crustacea: Estomatopoda, Gonodactylidae y Pseudosquillidae) An. Inst. Invest. Mar. P. Betín 18:95-112.

33. MURILLO, C.; ÁLVAREZ, R. 2004. Nuevos registros y consideraciones biogeográficas de los estomatópodos (Hoplocarida: Stomatopoda) del Pacífico colombiano y comparación con las especies del Caribe colombiano. En: Hendrickx, M.E. ed. Contribuciones al estudio de los crustáceos del Pacífico este. Inst. Cien. Mar Limol. UNAM, México, D.F. 3:1-16.

34. NAVIA, A.; GIRALDO, A.; MEJÍA, P. 2006. Nota sobre la biología del toyo vieja (Mustelus lunulatus) en 
la zona central de pesca del Pacífico colombiano. Invest. Mar., Valparaíso 34(2):217-222.

35. PINEDA, J.; HARE, J.; SPONAUGLE, S. 2007. Larval transport and dispersal in the coastal ocean and consequences for population connectivity. Oceanography. 20:22-39.

36. RAMÍREZ, V.; NIVIA, A.F.; RUBIO, E.A. 2008. Food habits and feeding ecology of an estuarine fish assemblage of northern Pacific Coast of Ecuador. Pan-Am. J.. Aquat. Sci. 3(3):361-372.

37. RAYMUNDO, A.; SAUCEDO, M. 2008. Hábitos alimentarios del pez tríglido Prionotus ruscarius (Gilbert \& Starks, 1904) durante 1996, en las costas de Jalisco y Colima, México. Rev. Bio. Mar. Oceanogr. 43(1):7-15.

38. REAKA, M.L. 1976. Lunar and tidal periodicity of molting and reproduction in stomatopod Crustacea: A selfish herd hypothesis. Biol. Bull. 150:468-490.

39. REAKA, M.L.; MANNING, R.B. 1987. The Significance of body size, dispersal potential, and habitat for rates of morphological evolution in stomatopod Crustacea. Smithson. Contr. Zool. 448:1-46.

40 REAKA, M.L.; RODGERS, P.J.; KUDLA, A.U. 2008. Patterns of biodiversity and endemism on IndoWest Pacific coral reefs. Proc. Natl. Acad. Sci. USA. 105(Suppl. 1):11474-11481.

41 RODRÍGUEZ, E.; SCHNEIDER, W. 2003. On the seasonal circulation within the Panama Bight derived from satellite observations of wind, altimetry and sea surface temperature. Geo. Res. Let. 30 (7):1410.

42 ROUX, A.; PIÑERO, R. 2006. Crustáceos decápodos y estomatópodos asociados a la pesquería del langostino patagónico Pleoticus muelleri (Bate, 1888) en el golfo de San Jorge, Argentina. Rev. Invest. Des. Pesq. 18:33-43.
43. SIEGEL, D.A.; MITARAI, S.; COSTELLO, C.J.; GAINES, S.D.; KENDALL, B.E.; WARNER, R.; WINTERS, K.B. 2008. The stochastic nature of larval connectivity among nearshore marine populations. Proc. Natl. Acad. Sci. USA 105:8974-8979.

44. SUTHERS, I.M.; RISSIK, D. 2009. Plankton: A guide to their ecology and monitoring for water quality. Com. Sci. Ind. Res. Org. (CSIRO) Pub., Collingwood. 272p.

45. SPONAUGLE, S.; COWEN, R.K.; SHANKS, A.; MORGAN, S.G.; LEIS, J.M.; PINEDA, J.; BOEHLERT, G.W.; KINGSFORD, M.J.; LINDEMAN, K.C.; GRIMES, C; MUNRO, J.L. 2002. Predicting self-recruitment in marine populations: Biophysical correlates and mechanisms. Bull. Mar. Sci. 70(1):341-375.

46. TANG, R.W.K.; YAU, C.; NG, W.C. 2010. Identification of stomatopod larvae (Crustacea: Stomatopoda) from Hong Kong waters using DNA barcodes. Mol. Eco. Res. 10(3):439-448.

47. VALADEZ, C.; AGUILAR, B.; HERNÁNDEZ, S. 2001. Hábitos alimentarios de la raya Urobatis halleri (Cooper, 1863) (Chondrichthyes: Urolophidae) capturada en la plataforma continental de Jalisco y Colima, México. Cienc. Mar. 27(1):91-104.

48. VILA, Y.; SOBRINO, I; JIMÉNEZ, M.P. 2013. Fishery and life history of spot-tail mantis shrimp, Squilla mantis (Crustacea: Stomatopoda), in the Gulf of Cadiz (eastern central Atlantic). Sci. Mar. 77(1):137-148.

49. VILLEGAS, N. 2003. Evolución mensual de las corrientes verticales y zonas de surgencia en la Cuenca del $\mathrm{Pa}$ cífico Colombiano. Bol. Cient. CCCP 9:34-44.

50. VINUESA, J.H. 2005. Distribución de crustáceos decápodos y estomatópodos del golfo San Jorge, Argentina. Rev. Biol. Mar. Oceanogr. 40(1):7-21

Recibido: Enero 5 de 2014

Aceptado: Abril 17 de 2014

Como citar:

López, R.H.; Jaimes, J.C. 2014. Aspectos de la distribución larval de Stomatopoda (Crustacea) en aguas superficiales del Pacífico colombiano. Rev. U.D.C.A Act. \& Div. Cient. 17(1): 227-236. 